\title{
Development of a voiding diary using urination recognition technology in mobile environment
}

\author{
Gun Hyun Park', Su Jin Kim², Young Sam Cho ${ }^{3, *}$ \\ 'Department of Urology, Gachon University Gil Medical Center, Gachon University College of Medicine, Incheon, Korea \\ 2Department of Urology, Yonsei University Wonju College of Medicine, Wonju, Korea \\ ${ }^{3}$ Department of Urology, Kangbuk Samsung Hospital, Sungkyunkwan University School of Medicine, Seoul, Korea
}

We invented a wearable device that can measure voiding time and frequency by checking a habitual series of characteristic motions among men. This study collected and analyzed urination time data collected smart bands worn by patients to resolve the clinical issues posed by using voiding charts. By developing a smart band-based algorithm for assessing urination time in patients, this study aimed to explore the feasibility of urination management systems. This study aimed to assess urination time based on a patient's posture and changes in posture. Motion data were obtained from a smart band on the arm. An algorithm that identifies the three stages of urination (forward movement, urination, backward movement) was developed based on data collected from a 3-axis accelerometer and tilt angle data. Therefore, we analyze hidden Markov model (HMM)-based sequential data to determine uri- nation time. Real-time data were acquired from the smart band. For data corresponding to a specific duration, the value of the signals was calculated and then compared with the set analysis model to calculate the time of urination. The final accuracy of the algorithm was calculated based on clinical guidelines for urologists. The experiment showed a high average accuracy of $92.5 \%$, proving the robustness of the proposed algorithm. The proposed urination time recognition technology draws on acceleration data and tilt angle data collected via a smart band; these data were then analyzed using a classifier after applying the HMM method.

Keywords: Urination time recognition, Urination management system, Mobile voiding chart

\section{INTRODUCTION}

Voiding problems are a common condition observed among elderly individuals, and voiding dysfunction is a significant risk factor for increased mortality and decreased quality of life among these individuals (Nakagawa et al., 2010; Pesonen et al., 2020; Wehrberger et al., 2012). For the evaluation of voiding dysfunction, diagnostic tools such as patient-reported symptom questionnaires, voiding diaries, and urodynamic studies are used in clinics. The voiding diary is a useful method to show the patient's real-life voiding habits and patterns. Therefore, in many lower urinary tract diseases such as overactive bladder and benign prostate hyperplasia, voiding diary is included in the research method as an essential evaluation (Choi et al., 2018; Chuang et al., 2018; Hsiao et al., 2019; Lee et al., 2018; Yu et al., 2019).
In general, the patients record the frequency of voiding, each voiding volume, and other symptoms associated with urination in the standard form of voiding diary by themselves (Bright et al., 2012; Yap et al., 2007). Despite the benefit of a paper-type voiding diary, there are some limitations. According to the patient's characteristics, low adherence to the voiding diary reduces the reliability of a paper-type voiding diary. Some patients fail to record to record or lose their voiding diary before the visiting the clinic. In particular, older people with cognitive dysfunction and physical weakness are unable to record their voiding patterns in the diary.

Therefore, an electronic voiding diary was developed to overcome the limitation of a paper-type voiding diary, and various types of mobile applications have been released. Sussman et al. (2016) reported that electronic voiding diaries showed better compliance
${ }^{*}$ Corresponding author: Young Sam Cho (iD https://orcid.org/0000-0002-2966-7971 Department of Urology, Kangbuk Samsung Hospital, Sungkyunkwan University School of Medicine, 29 Saemunan-ro, Jongno-gu, Seoul 03181, Korea E-mail: choys1011@naver.com

Received: November 2, 2020 / Accepted: December 5, 2020
This is an Open Access article distributed under the terms of the Creative Commons Attribution Non-Commercial License (https://creativecommons.org/licenses/by-nc/4.0/) which permits unrestricted non-commercial use, distribution, and reproduction in any medium, provided the original work is properly cited. 
and data reliability than paper-based voiding diaries. Moreover, electronic voiding diaries seem to have merit because people are familiar with using smartphones and mobile devices at present. However, electronic voiding diaries may not apply to older people unfamiliar with mobile devices. Thus, a new method instead of conventional paper or electronic voiding diaries is necessary for the elderly population. To overcome the limitation of the previously developed electronic voiding diary, we evaluated the feasibility of a new type of voiding diary based on recognition technology monitoring patient behavior.

\section{MATERIALS AND METHODS}

This study was included healthy men aged $\geq 18$ years old and aimed to evaluate the efficacy of a new type of voiding diary based on recognition technology. The mean age was 28.73 (range, 2634) years old, and none of the patients showed voiding dysfunction. The patients completed a 3-day paper voiding diary and wore a smart band to monitor the number of urinations. The smart band used in this study is able to detect urination based on a patient's posture and changes in posture. Motion data were obtained from a smart band worn on the arm. An algorithm that recognizes the three stages of urination (forward movement, urination, backward movement) was developed based on data collected from a 3-axis accelerometer and tilt angle data. The feature used for the analysis of sequential data has temporal characteristic. Therefore, we analyzed hidden Markov model (HMM)-based sequential data to detect urination. Real-time data were acquired from the smart band, and for data corresponding to a certain duration, the value of the signals was calculated and then compared with the set analysis model to determine the time of urination. The paper voiding diary was reviewed by two urologists, and the date reported from the smart band was compared with the results of the paper voiding diary. This study was conducted in accordance with the Declaration of Helsinki and approved by the Institutional Review Board of the Gachon University Gil Medical Center (GDIRB2017-096). All patients provided written informed consent.

\section{User-adaptable postprocessing model}

The following is a user-adaptable postprocessing model to increase the accuracy of HMM recognition results. The entire process extracts feature from the entered signals and performs the primary recognition process using HMM. User-adaptable postprocessing is performed by receiving the results from the device to check the application of postprocessing, and if postprocessing is required, the final results are derived. Each node in the input layer is entered with Min-max Normalization of the recognizable urinary signal, and each node in the output layer is modified into a class to be modified by processing. In other words, in the case of a misrecognized case, the case is collected to create a processing model, and if the same results are obtained at the next treatment, the case is corrected. Therefore, the accuracy of urination activity recognition was improved to user-tailored form and nodes to self-adaptive type.

\section{Statistical analysis}

Based on a total of 15 men cases, measure normal detection (true positive; TP), false detection (false positive; FP), and detection failure (false negative; FN) by comparing the number of manually written urination with the number of actual recognized waveforms. Sensitivity and specificity are calculated from measured TP, FP, and FN information to confirm the effectiveness and accuracy of the proposed urination recognition technology. The data were presented in terms of the mean values of continuous variables and the frequencies of categorical variables. For diagnostic test performance, the number of patient's outcomes with a TP, FP, FN, and true negative test result were extracted. The primary objective was to estimate the sensitivity and specificity with 95\% confidence interval (CI), and diagnostic odds ratio (DOR) with $95 \%$ CI. A DOR can be calculated as the ratio of the odds of probability in a urination state relative to the odds of probability in the non-urination state, with higher values indicating better discriminatory test performance (Glas et al., 2003). Betweenindividual statistical heterogeneity was assessed using $\mathrm{I}^{2}$ and the Cochrane $\mathrm{Q}$ test on the basis of the random-effects analysis. The bivariate model estimates pairs of logit transformed sensitivity and specificity, incorporating the correlation that might exist between sensitivity and specificity (Thompson, 1994). We used the bivariate random-effects model for analysis and pooling of the diagnostic performance measures across patient's outcomes (Reitsma et al., 2005). We also constructed hierarchical summary receiver operator characteristic (HSROC) curves to overcome some limitations of the traditional summary ROC curve procedure (Shim et al., 2019). Each data point of the HSROC graph comes from an individual patient's outcome; then, the HSROC curve is formed based on these points to form a smooth curve to reveal pooled accuracy. Two-sided $P \leq 0.05$ was considered statistically significant. 

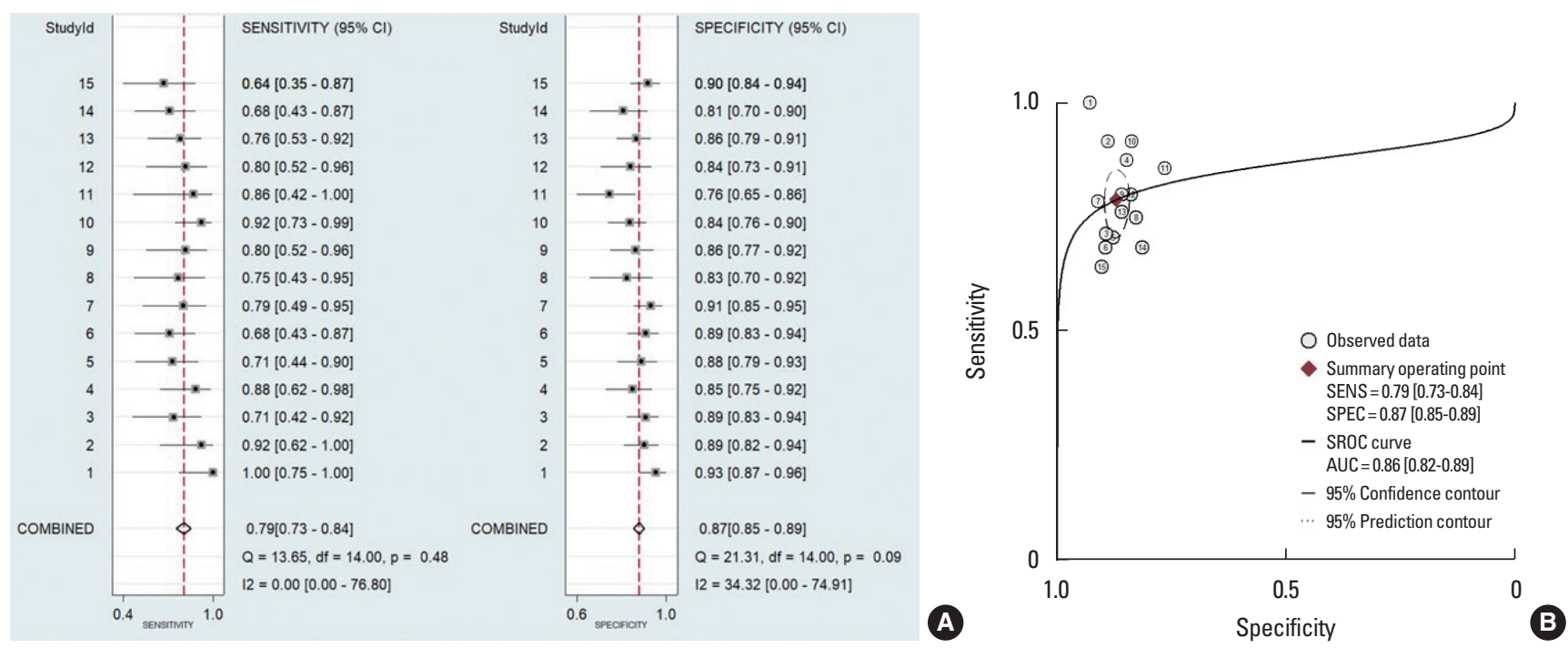

Fig. 1. Sensitivity and specificity of recognition technology monitoring urination. (A) Each participant data. (B) The receiver operating curve. Cl, confidence interval; AUC, area under the curve; SENS, sensitivity; SPEC, specificity.

Table 1. Overall motion data $(n=15)$

\begin{tabular}{lccc}
\hline Variable & Coefficient & Standard error & 95\% Confidence interval \\
\hline Sensitivity & 0.789 & 0.0269 & $0.731-0.837$ \\
Specificity & 0.8709 & 0.010 & $0.850-0.889$ \\
DOR & 25.031 & 4.517 & $17.574-35.657$ \\
LR+ & 6.0734 & 0.498 & $5.172-7.132$ \\
LR- & 0.2426 & 0.310 & $0.1890-0.312$ \\
1/LR- & 4.121 & 0.526 & $3.210-5.292$ \\
\hline
\end{tabular}

DOR, diagnostic odds ratio; LR+, positive likelihood ratio; LR-, negative likelihood ratio.

\section{RESULTS}

We evaluated the efficacy of using a smart band to monitor urination in 15 men without a voiding problem. The DOR of urination reported from the smart band was 25.03 (95\% CI, 17.5735.65). Heterogeneity between the participants was not observed $(P=0.839)$ (Table 1). An analysis of smart band-monitored urination showed that smart band can be used to reliably and accurately recognize human behavior patterns using smart band. Fig. 1 showed that the pooled sensitivity and specificity were $0.79(95 \%$ CI, 0.73-0.84) and 0.87 (95\% CI, 0.85-0.89) respectively.

\section{DISCUSSION}

This is the clinical study to show the efficacy of using a mobile device to monitor urination in real-life. We proposed the use of a smart band to monitor urination behavior using motion recognition technology and developed an algorithm based on the characteristic movement commonly observed in men during urination. We chose HMM for the algorithm to recognize urination behavior because HMM is more suitable for analyzing sequential data that have temporal characteristics than other algorithms such as Bayesian networks, HMMs, and recurrent neural networks (LeCun et al., 2015). We confirmed that the frequency of urinations recorded by the smart band loaded with the urination monitoring device proposed in this study was not different from the results in the conventional paper voiding diary.

Many studies on the application of mobile health have been performed in various medical fields. In the urologic area, there have been many attempts to develop mobile medical devices for both patients and clinicians (Hwang, 2018; Kang et al., 2018). Voiding dysfunction from various urologic diseases is prevalent and associated with low quality of life because voiding problems restrict daily life (Egan, 2016; Yoo et al., 2018). At present, most of the examination for voiding dysfunction is performed in the clinic; therefore, the test does not represent the patient's real-life urination. Moreover, the patients cannot express their symptoms by only one examination in the hospital. Therefore, a voiding diary is used to assess the patient's urination pattern in real life. The patient records the number of urination, urination, volume, or other symptoms associated with urination in the specialized paper for 3 days (Hsiao et al., 2019). The findings of the voiding diary provide information on urination in real life because the patients 
record it during their everyday activity. However, some patients cannot record at every urination, and some of them lost their paper voiding diary before visiting the hospital. Thus, there have been many studies that examined the use of mobile devices to overcome the challenges of paper voiding diaries. A study by Vaccari et al. (2020) reported that there were many types of mobile voiding diaries that could be used in place of the paper voiding diaries. The use of an electronic mobile voiding diary seems to increase adherence compared with paper voiding diaries because people are familiar with the mobile devices (Abrams et al., 2016).

Although electronic and mobile voiding diaries have shown some benefits, there are still limitations. Previous studies that evaluated mobile medical applications or devices were performed using individuals who were familiar with the mobile device. The electronic voiding diary used at present is not suitable for older adults who do not use the mobile device due to cognitive problems or frailty (Wildenbos et al., 2018). Therefore, a new method to monitor real-life urination patterns is necessary for these dependent and frail older people. We used recognition technology to monitor human behavior to overcome the electronic voiding diary. We reported the reliability of the algorithm focused on men's characteristic movement during urination (Eun et al., 2017; Whangbo et al., 2018).

Moreover, the possibility of a smart band with a recognition system was tested in a previous pilot study (Chung et al., 2019). Based on these previous studies, a smart band for real users was developed, and the accuracy of this technology was tested in the present study. Compared with paper voiding diaries, the data recorded by the smart band loaded with the motion recognition technology were reliable because the sensitivity and specificity were high. As a result, the new mobile voiding diary can be a useful method to monitor urination behavior and diagnose voiding problems in men. Moreover, our new method can detect and record urination behavior by itself. Therefore, it can be a better option for dependent older people.

Despite the benefits of mobile methods compared with paper and electronic voiding diaries, there are some limitations. The smart band developed in this study was only for men. Thus, we have been studying to find characteristic urination movements and establish mobile devices for women. This study included men without voiding problems who were familiar with the mobile device. Therefore, further studies are planned to be performed in patients with voiding dysfunction and elderly individuals with cognitive issues. Voiding diaries based on urination movement recognition technology can be a useful option to monitor real-life uri- nation patterns in men.

\section{CONFLICT OF INTEREST}

No potential conflict of interest relevant to this article was reported.

\section{ACKNOWLEDGMENTS}

This work was supported by research grants funded by the RIMHealthcare, Inc.

\section{REFERENCES}

Abrams P, Paty J, Martina R, Newgreen DT, van Maanen R, Paireddy A, Kuipers-deGroot T, Ridder A. Electronic bladder diaries of differing duration versus a paper diary for data collection in overactive bladder. Neurourol Urodyn 2016;35:743-749.

Bright E, Cotterill N, Drake M, Abrams P. Developing a validated urinary diary: phase 1. Neurourol Urodyn 2012;31:625-633.

Choi H, Bae JH, Oh CY, Jeong SJ, Ko WJ, Choi JB, Seo JT, Lee DH, Kim JC, Lee KW, Kim YH. Clinical efficacy of solifenacin in the management of diabetes mellitus-associated versus idiopathic overactive bladder symptoms: a multicenter prospective study. Int Neurourol J 2018;22: 51-57.

Chuang FC, Hsiao SM, Kuo HC. The overactive bladder symptom score, international prostate symptom score-storage subscore, and urgency severity score in patients with overactive bladder and hypersensitive bladder: Which scoring system is best? Int Neurourol J 2018;22:99-106.

Chung KJ, Kim J, Whangbo TK, Kim KH. The prospect of a new smart healthcare system: a wearable device-based complex structure of position detecting and location recognition system. Int Neurourol J 2019; 23:180-184.

Egan KB. The epidemiology of benign prostatic hyperplasia associated with lower urinary tract symptoms: prevalence and incident rates. Urol Clin North Am 2016;43:289-297.

Eun SJ, Whangbo TK, Park DK, Kim KH. Development of personalized urination recognition technology using smart bands. Int Neurourol J 2017;21:S76-83

Glas AS, Lijmer JG, Prins MH, Bonsel GJ, Bossuyt PM. The diagnostic odds ratio: a single indicator of test performance. J Clin Epidemiol 2003;56: 1129-1135.

Hsiao SM, Wu PC, Chang TC, Chen CH, Lin HH. Urodynamic and bladder diary factors predict overactive bladder-wet in women: a comparison with overactive bladder-dry. Int Neurourol J 2019;23:69-74. 
Hwang YS. Study on the voiding detection system using wearable device. Int Neurourol J 2018;22:S65-65.

Kang M, Park E, Cho BH, Lee KS. Recent patient health monitoring platforms incorporating internet of things-enabled smart devices. Int Neurourol J 2018;22:S76-82.

LeCun Y, Bengio Y, Hinton G. Deep learning. Nature 2015;521:436-444. Lee YS, Lee KS, Choo MS, Kim JC, Lee JG, Seo JT, Lee JZ, Lee JY, Oh SJ, $\mathrm{Na}$ YG. Efficacy of an alpha-blocker for the treatment of nonneurogenic voiding dysfunction in women: an 8-week, randomized, double-blind, placebo-controlled trial. Int Neurourol J 2018;22:30-40.

Nakagawa H, Niu K, Hozawa A, Ikeda Y, Kaiho Y, Ohmori-Matsuda K, Nakaya N, Kuriyama S, Ebihara S, Nagatomi R, Tsuji I, Arai Y. Impact of nocturia on bone fracture and mortality in older individuals: a Japanese longitudinal cohort study. J Urol 2010;184:1413-1418.

Pesonen JS, Cartwright R, Vernooij RWM, Aoki Y, Agarwal A, Mangera A, Markland AD, Tsui JF, Santti H, Griebling TL, Pryalukhin AE, Riikonen J, Tähtinen RM, Vaughan CP, Johnson TM, 2nd, Auvinen A, Heels-Ansdell D, Guyatt GH, Tikkinen KAO. The impact of nocturia on mortality: a systematic review and meta-analysis. J Urol 2020;203: 486-495.

Reitsma JB, Glas AS, Rutjes AW, Scholten RJ, Bossuyt PM, Zwinderman $\mathrm{AH}$. Bivariate analysis of sensitivity and specificity produces informative summary measures in diagnostic reviews. J Clin Epidemiol 2005; 58:982-990.

Shim SR, Kim SJ, Lee J. Diagnostic test accuracy: application and practice using R software. Epidemiol Health 2019;41:e2019007.

Sussman RD, Richter LA, Tefera E, Park AJ, Sokol AI, Gutman RE, Iglesia CB. Utilizing technology in assessment of lower urinary tract symp- toms: a randomized trial of electronic versus paper voiding diaries. Female Pelvic Med Reconstr Surg 2016;22:224-228.

Thompson SG. Why sources of heterogeneity in meta-analysis should be investigated. BMJ 1994;309:1351-1355.

Vaccari NA, da Silveira LTY, Bortolini MAT, Haddad JM, Baracat EC, Ferreira EAG. Content and functionality features of voiding diary applications for mobile devices in Brazil: a descriptive analysis. Int Urogynecol J 2020;31:2573-2581.

Wehrberger C, Madersbacher S, Jungwirth S, Fischer P, Tragl KH. Lower urinary tract symptoms and urinary incontinence in a geriatric cohort - a population-based analysis. BJU Int 2012;110:1516-1521.

Whangbo TK, Eun SJ, Jung EY, Park DK, Kim SJ, Kim CH, Chung KJ, Kim $\mathrm{KH}$. Personalized urination activity recognition based on a recurrent neural network using smart band. Int Neurourol J 2018;22:S91-100.

Wildenbos GA, Peute L, Jaspers M. Aging barriers influencing mobile health usability for older adults: A literature based framework (MOLDUS). Int J Med Inform 2018;114:66-75.

Yap TL, Cromwell DA, Brown C, Emberton M, van der Meulen J. The reliability of the frequency-volume chart in assessing lower urinary tract symptoms. BJU Int 2007;100:111-115.

Yoo TK, Lee KS, Sumarsono B, Kim ST, Kim HJ, Lee HC, Kim SH. The prevalence of lower urinary tract symptoms in population aged 40 years or over, in South Korea. Investig Clin Urol 2018;59:166-176.

Yu HS, Lee JW, Yu J, Cho MC, Cho SY. A prospective, multicenter, openlabel study of dose escalation therapy in male patients with nocturia refractory to 0.2-mg tamsulosin monotherapy. Int Neurourol J 2019; 23:294-301. 